\title{
The Role of Prescribing Safety Assessment on Prescribing Readiness of Medical Students in Malaysia
}

\author{
Thuraiveloo, S. ${ }^{1}$, Pillai, S. ${ }^{1}$, Karunakaran, L.G. ${ }^{1}$, Mui, S.S. ${ }^{2}$, Maxwell, S. ${ }^{3}$, \\ Mathialagan, A.G. ${ }^{1}$ \& Karisnan, K. ${ }^{1}$
}

\begin{abstract}
Introduction: Many interventions in terms of training and curriculum enhancement have been introduced to reduce prescribing errors among doctors. One such initiative is the introduction of Prescribing Safety Assessment (PSA) which is a learning module that enhances pharmacological and therapeutics knowledge. However, the benefits of PSA have not been evaluated in Malaysian context before. This study aimed to determine the role of the PSA module on self-perceived prescribing knowledge and competency among medical students.
\end{abstract}

Methods: A cross-sectional comparative study design was used in which two groups of medical students from different cohorts were asked to fill in an online questionnaire regarding their competency and confidence in the area of prescribing. These groups consisted of 5th year medical students who had undergone the PSA module $(n=52)$ and who did not have any exposure to PSA module $(n=54)$. Independent T-test was used to compare the mean values of answers for each of the questions from both cohorts to determine significance.

Results: The mean data showed that the respondents from both groups tended to 'agree' to have sufficient prescribing knowledge in drugs. Respondents from both groups generally agreed to be confident in prescribing skills.

Conclusion: The respondents in our study generally perceive themselves to be both competent and confident in their prescribing skills. There was no obvious distinction between those who had undergone the PSA module and those who had not.

Key words: Prescribing competency and confidence, Medical doctors

\section{Introduction}

Several studies have shown that prescribing errors among doctors are multifactorial but can be grouped into two main aspects which are patient-related and work environment related (Dean, 2002; Ryan et al., 2014).

\footnotetext{
${ }^{1}$ School of Medicine, Perdana University-RCSI, Malaysia.

${ }^{2}$ Department of Pharmacology, Faculty of Medicine, University of Malaya, Malaysia.

${ }^{3}$ Clinical Research Centre, University of Edinburgh, Western General Hospital, Edinburgh, UK.
}

Corresponding Author: Kanakeswary Karisnan

Perdana University, Block $B$ and D1, MAEPS Building, MARDI Complex Jalan MAEPS Perdana, 43400 Seri Kembangan, Selangor, Malaysia.

Email: kanakeswary@perdanauniversity.edu.my
The above studies also report that the majority of prescribing errors were due to the working environment and extensive workload followed by individual factors. Among the key individual factors are lack of pharmacology knowledge, prescribing knowledge and experience (Hansen et al., 2016; Coombes et al., 2008). These findings are of major concern as they indicate that there is an issue in the overall prescribing preparedness of students as they graduate from medical school. Although it is agreeable that most experience can only be attained during clinical practice as a doctor, simulated prescribing scenarios introduced throughout the medical course can be helpful in improving students' prescribing preparedness.

Prescribing errors have become a global issue (Van Doormaal et al., 2009; Dean et al., 2002; Tully, 2012) and they are increasingly evident

DOI: http://doi.org/10.4038/seajme.v14i1.233

(C) 1 SEAJME. This is an Open Access article distributed under the terms of the Creative Commons Attribution
License (http://creativecommons.org/licenses/by/4.0/), which permits unrestricted use, distribution, and
reproduction in any medium, provided the original author and source are credited


in Malaysian hospitals and clinics as well (Kuan et al., 2002; Rahman et al., 1994; Abdullah et al., 2004). A study done to determine medication errors among geriatrics found that $25 \%$ of the prescriptions had medication errors (Abdullah et al., 2004). To corroborate, 60.4\% of hospital pharmacists perceive that prescribing errors by doctors are common and $25.6 \%$ of them thought that the prescribing errors were due to lack of knowledge and carelessness (Rahman et al.,1994). Another study on paediatric in-patient prescribing errors in Malaysia identified lack of supervision and lack of knowledge as most common contributing factors (Khoo et al., 2017). These are significant findings which should be given due consideration as medication errors could lead to increased morbidity and mortality in patients. Apart from the disease burden, these errors would also contribute towards an increase in healthcare costs for patients as well as healthcare professionals. It is clear that these iatrogenic complications could be easily prevented if the doctors were more selfequipped with the necessary skills and knowledge required to safely prescribe drugs.

As a remedy for the increasing prevalence of prescribing errors among doctors, the British Pharmacological Society together with the Medical Schools Council Assessment (MSCA) developed the Prescribing Safety Assessment (PSA) (Maxwell et al., 2015). PSA assesses the prescribing skills of final-year medical students and is based on competencies identified by the General Medical Council. Those competencies are writing new prescriptions, reviewing existing prescriptions, calculating drug doses, identifying and avoiding both adverse drug reactions and medication errors and amending prescribing to suit individual patient circumstances. The PSA is an online openbook assessment that encompasses eight different sections which reflect on different aspects of the prescribing process; 1. Prescribing; 2. Prescription review; 3. Planning management; 4. Providing information about medicines; 5 . Calculation skills; 6 . Adverse drug reactions; 7 . Drug monitoring and 8 . Data interpretation.

Overall, the PSA serves as an assessment of skills and knowledge in prescribing which allows medical students to showcase their confidence and competence to prescribe medication. There is evidence to show that the reliability of the assessment and performance in the assessment is improving (Maxwell et al., 2017), however research in this area is limited. Thus, the aim of this study was to examine the role of a PSA module on self-perceived competency and confidence to prescribe among final year medical students at Perdana University-Royal College of Surgeons in Ireland (PU-RCSI).

\section{Methods}

The objective of the study was to determine the effect of prescribing module content towards medical students' perception towards prescribing competently and confidently.

\section{Study Design and Population}

A cross sectional comparative study was designed to meet the study objectives. The study population comprised of final year medical students from Perdana UniversityRoyal College of Surgeons in Ireland (PURCSI). The respondents were included based on the inclusion criteria for two specific groups, being year 5 medical students from graduating cohort 2019 who have had a compulsory PSA module and the year 5 medical students from graduating cohort 2018 who did not undergo the PSA module. The PU-RCSI curriculum used the same PSA module but did not use the same form of assessment as per the British Pharmacological Society. The assessment had a list of questions which were designed to suit the healthcare practice in Malaysia. However it still encompassed the eight different aspects of the prescribing process which is in line with the standards of the British Pharmacological Society and the Medical Schools Council Assessment.

\section{Study Instrument}

The study instrument was developed based on an existing questionnaire to obtain students' views on their personal prescribing skills and competencies (Heaton et al., 2008). The questionnaire consisted of 28 questions in total with 12 being multiple choice questions and 16 being Likert scale questions (Supplementary Table 1). The questions revolved around three main areas; basic pharmacology and therapeutics, prescribing skills and competencies and prescribing confidence.

\section{Data Collection Procedure}

The web links of the questionnaires were sent via email to each of the cohorts. The responses were kept anonymous and inferred consent was obtained before respondents began answering the questionnaire. 


\section{Data Analysis}

The data was analysed using SPSS version 22 and was expressed as mean values. Independent t-test was used to determine the significance of difference between the mean values. Statistical analysis was performed at a $95 \%$ confidence interval and significance was determined at $p<0.05$.

This study was approved by Perdana University Institutional Review Board (PU IRBHR0179).

Table 1: Mean values of responses from pre-PSA and post-PSA 5th year medical students for questions regarding drug prescribing knowledge

\begin{tabular}{|c|c|c|c|}
\hline \multirow{2}{*}{ Drugs } & \multicolumn{2}{|c|}{ Mean ( \pm SEM) values } & \multirow[t]{2}{*}{ p-value } \\
\hline & pre-PSA & post-PSA & \\
\hline Analgesia/NSAID & $4.09 \pm 0.09$ & $4.00 \pm 0.10$ & 0.478 \\
\hline Opiates & $3.83 \pm 0.10$ & $3.75 \pm 0.10$ & 0.550 \\
\hline Laxatives & $3.64 \pm 0.11$ & $3.73 \pm 0.10$ & 0.573 \\
\hline Antibiotics & $3.81 \pm 0.10$ & $3.61 \pm 0.11$ & 0.162 \\
\hline Anti-Emetics & $3.89 \pm 0.10$ & $3.86 \pm 0.10$ & 0.860 \\
\hline Anti-Hypertensives & $4.17 \pm 0.06$ & $4.12 \pm 0.11$ & 0.691 \\
\hline Anti-Diabetes & $4.19 \pm 0.07$ & $4.20 \pm 0.10$ & 0.929 \\
\hline Anti-Coagulants & $3.92 \pm 0.11$ & $3.78 \pm 0.10$ & 0.337 \\
\hline Anti-Histamines & $3.78 \pm 0.11$ & $3.74 \pm 0.11$ & 0.843 \\
\hline Anti-Asthmatics & $4.17 \pm 0.07$ & $4.08 \pm 0.10$ & 0.454 \\
\hline Peptic ulcer medication & $4.17 \pm 0.09$ & $4.10 \pm 0.10$ & 0.615 \\
\hline
\end{tabular}

Statistical procedure: independent $t$-Test (significant level at $p<0.05)$

Pre-PSA $(n=54)$ and Post PSA $(n=52)$

\section{Results}

A total of 106 responses were obtained which gave a response rate of $77 \%$. Of the 106 respondents, 54 comprised of 5 th year medical students who did not undergo the PSA module while the other 52 were 5 th year medical students who underwent the PSA module. All 106 respondents described their medical course as 'integrated'. Here on, the 5th year medical students who did not undergo PSA will be referred as Pre-PSA while the 5th year medical students who did undergo PSA will be referred to as Post-PSA.

\section{Validity and Reliability}

The questionnaire was tested for internal consistency using Cronbach alpha where a score of 0.897 was recorded, indicating a good reliability. In terms of validity, authors conducted a face validity test to ensure the items were measuring the desired objectives. To measure construct validity, the instrument had undergone factor analysis where it was 
observed that 26 of the 28 items correlated at least 0.3 with at least one other item, suggesting reasonable factorability. Secondly, the Kaiser-Meyer-Olkin measure of sampling adequacy was 0.81 , above the commonly recommended value of 0.6 , and the Bartlett's test of sphericity was significant. The diagonals of the anti-image correlation matrix were all over 0.5. Finally, the communalities were all above 0.4 , further confirming that each item shared some common variance with other items. Given these overall indicators, factor analysis was deemed to be suitable for all items concerned.

Learning of Basic Pharmacology and Clinical Pharmacology and Therapeutics

The respondents identified their learning style in Basic Pharmacology as well as Clinical Pharmacology and Therapeutics to be 'mainly integrated learning system-based modules'. When asked about the professionals that have played a significant role in teaching them about drug prescribing, a majority (57\%) of pre-PSA 5th year medical students responded as clinicians and $24 \%$ answered as general practitioners. As for the post-PSA 5th year medical students, clinicians have played a greater role in teaching them about drug prescribing than general practitioners with $70 \%$ indicating the former and only $16 \%$ indicating the latter.

\section{Competency to Prescribe}

Table 1 shows the mean values of responses from both the cohorts for questions regarding drug prescribing knowledge. When asked about the students' views on whether they believe that they have sufficient knowledge in prescribing common drugs, the mean values of the cohorts showed no significant difference. Both the cohorts 'agree' that they have sufficient knowledge in prescribing common drugs.

\section{Confidence to Prescribe}

Table 2 shows the mean values of responses from pre-PSA and post-PSA 5th year medical students for questions regarding confidence in prescribing skills. With regards to having confidence in prescribing skills, the two cohorts did not show any significant difference as both cohorts tended to 'agree' on being confident in these areas of prescribing except for drug dose calculation $(p=0.034)$. However both cohorts on average responded 'uncertain'.

Table 2: Mean values of responses from pre-PSA and post-PSA 5th year medical students for questions regarding confidence in prescribing skills

\begin{tabular}{|c|c|c|c|}
\hline \multirow{2}{*}{ Prescribing skills } & \multicolumn{2}{|c|}{ Mean ( \pm SEM) values } & \multirow[t]{2}{*}{ p-value } \\
\hline & pre-PSA & post-PSA & \\
\hline Drug history taking & $4.22 \pm 0.08$ & $4.12 \pm 0.10$ & 0.418 \\
\hline Prescription writing & $3.65 \pm 0.09$ & $3.82 \pm 0.10$ & 0.203 \\
\hline Reviewing patient medication charts & $3.93 \pm 0.09$ & $3.66 \pm 0.12$ & 0.078 \\
\hline Drug dosage calculation & $3.39 \pm 0.11$ & $3.02 \pm 0.13$ & $0.034^{*}$ \\
\hline Accessing drug information & $3.84 \pm 0.08$ & $3.94 \pm 0.09$ & 0.401 \\
\hline
\end{tabular}

Statistical procedure: independent $t$-Test (significant level at $p<0.05)$, *significantly different Pre-PSA $(n=54)$ and Post-PSA $(n=52)$ 


\section{Discussion}

The current study aimed to identify the role of PSA on self-perceived competency and confidence to prescribing drugs by final year medical students. Results showed that there were no significant differences between the pre-PSA and post-PSA cohorts. This indicates that both the cohorts had similar degree of selfperceived confidence and competency although one cohort was exposed to PSA training and evaluation.

This finding is interesting as it implies that the graduates perceived that they are provided adequate training within the medical school curriculum with or without PSA. The RCSI curriculum places high emphasis on prescribing competency and pharmacological knowledge. As opposed to other medical curricula (Ramasamy \& Osman, 2005; Lim, 2012), the students are exposed to the importance of safe prescribing and dangers of medication errors from Year one. Students are also strenuously trained on the distinct pharmacological and therapeutic properties of each drug category across the curriculum from Year one to Year five. Additionally, the RCSI curriculum engages with both hospital and general practitioner clinics to ensure that students have been exposed to numerous prescriptions in a real world setting that increases confidence and competency. According to a study, teaching methods and educational interventions on prescribing would be able to effectively enhance the students' knowledge and skills in prescribing and consequently produce competent prescribers (Kamaruddin et al., 2013). Similarly, prescribing simulation exercises are important tools in developing prescribing and therapeutics skills (Rothwell et al., 2012; Ross \& Maxwell 2012; Maxwell, 2012). Importantly, a previous study by RCSI indicates that the students who had undergone a four week intern-training programme which covered clinical and technical skills as well as a four day shadowing experience showed a rise in prescribing readiness and their readiness on undertaking the internship year (Gouda, 2016). The medical curriculum of PU-RCSI also affords a high exposure to evaluations such as OSCEs which provide them with simulated situations where their prescribing skills can be put to the test. This creates strong impetus for the students to gain the same prescribing skills and competency similar to PSA. This explains the similarities in competency, confidence and knowledge among those with PSA training and non-PSA training.

\section{Limitations}

This study has two notable limitations. Firstly, the measures utilized in this study were selfreported as opposed to actual evaluated competencies. It is generally an accepted fact that discrepancies do occur between selfreported data and actual evaluation results (Brinkman et al., 2015). Hence, this result should be interpreted with caution as actual competence of the respondents was not assessed. Nonetheless, such an exploration into the possible role of PSA in medical training in Malaysia has not been attempted before and as such this study contributes to the available body of knowledge. Secondly, the respondents of this study were obtained from a high tier medical school and the role of PSA may be different in other medical schools in Malaysia.

\section{Conclusion and Recommendations}

In conclusion, our study showed that there is no difference between pre-PSA and post-PSA cohorts, on self-perceived prescribing competence and confidence, indicating that the existing prescribing education and training in this particular medical school may be adequate to provide confidence and competency for medical students in embarking on their medical career. Nevertheless, further studies that involve multiple schools with varying curricula and an objective assessment to explore prescribing readiness and impact of PSA are required to confirm the above conclusion.

\section{Conflict of Interest}

The authors declare no conflict of interest.

\section{Acknowledgements}

We would like to thank all participants. This research was supported by Perdana University, Royal College of Surgeons in Ireland summer research funding.

\section{References}

Abdullah, D.C., Ibrahim, N.S. \& Ibrahim, M.I.M. (2004) Medication Errors among Geriatrics at the Outpatient Pharmacy in a Teaching Hospital in Kelantan, The Malaysian Journal of Medical Sciences: MJMS, 11, 2, pp.52.

Brinkman, D.J., Tichelaar, J., van Agtmael, M.A., de Vries, T.P. \& Richir, M.C. (2015) Self-reported Confidence in Prescribing Skills Correlates Poorly with Assessed Competence in FourthYear Medical Students, The Journal of Clinical Pharmacology, 55, 7, pp.825-830. 
Coombes, I.D., Stowasser, D.A., Coombes, J.A. \& Mitchell, C. (2008) Why Do Interns Make Prescribing Errors? A Qualitative Study, Medical Journal of Australia, 188, 2, pp. 89-94.

Dean, B., Schachter, M., Vincent, C. \& Barber, N. (2002) Causes of Prescribing Errors in Hospital Inpatients: A Prospective Study, The Lancet, 359, 9315, pp.1373-1378.

Dean, B., Schachter, M., Vincent, C. \& Barber, N. (2002) Prescribing Errors in Hospital Inpatients: Their Incidence and Clinical Significance, Quality and Safety in Health Care, 11, 4, pp.340-344.

Gouda, P., Kitt, K., Evans, D.S., Goggin, D., McGrath, D., Last, J., Hennessy, M., Arnett, R., O'Flynn, S., Dunne, F. \& O'Donovan, O.D. (2016) Irish Medical Students' Understanding of the Intern Year, Irish Medical Journal, 109, 4:387.

Hansen R, Bradley CP, \& Sahm, L.J. (2016) Factors Influencing Successful Prescribing by Intern Doctors: A Qualitative Systematic Review, Pharmacy, 4, 3, p.24.

Heaton, A., Webb, D.J. \& Maxwell, S.R. (2008) Undergraduate Preparation for Prescribing: The Views of 2413 UK Medical Students and Recent Graduates, British Journal of Clinical Pharmacology, 66, 1, pp.128-134.

Kamarudin, G., Penm, J., Chaar, B. \& Moles, R. (2013) Educational Interventions to Improve Prescribing Competency: A Systematic Review, BMJ Open, 3, 8, p.e003291.

Khoo, T.B., Tan, J.W., Ng, H.P., Choo, C.M. \& Teh, S.H. (2017) Paediatric In-patient Prescribing Errors in Malaysia: A Cross-Sectional Multicentre Study, International Journal of Clinical Pharmacy, 39, 3, pp. 551-559.

Lim, V.K.E. (2012) The Process of Medical Curriculum Development in Malaysia, International Journal of User-Driven Healthcare (IJUDH), 2, 1, pp.33-39.

Maxwell, S.R. (2012) An Agenda for UK Clinical Pharmacology: How Should Teaching of Undergraduates in Clinical Pharmacology and Therapeutics be Delivered and Assessed? British Journal of Clinical Pharmacology, 73, 6, pp. 893899.

Maxwell, S.R., Cameron, I.T. \& Webb, D.J. (2015) Prescribing Safety: Ensuring that New Graduates are Prepared, The Lancet, 385, 9968, pp. 579581.
Maxwell, S.R., Coleman, J.J., Bollington, L., Taylor, C. \& Webb, D.J. (2017) Prescribing Safety Assessment 2016: Delivery of a National Prescribing Assessment to 7343 UK Final-year Medical Students, British Journal of Clinical Pharmacology, 83, 10, pp. 2249-2258.

Ni, K.M., Siang, C.S. \& bin Ramli, M.N. (2002) Noncompliance with Prescription Writing Requirements and Prescribing Errors in an Outpatient Department, Age, 130, pp.32-7.

Rahman, A.R., Noor, A.R. \& Hassan, Y. (1994) How Good are Doctors as Drug Prescribers? Medical Journal of Malaysia, 49, 4, pp.364-368.

Ramasamy, P. \& Osman, A. (2005) The Medical School Curriculum at University Malaysia Sabah, The Medical Journal of Malaysia, 60, pp. 58-65.

Ross, S. \& Maxwell, S (2012) Prescribing and the Core Curriculum for Tomorrow's Doctors: BPS Curriculum in Clinical Pharmacology and Prescribing for Medical Students, British Journal of Clinical Pharmacology, 74, 4, pp.644-661.

Rothwell, C., Burford, B., Morrison, J., Morrow, G., Allen, M., Davies, C., Baldauf, B., Spencer, J., Johnson, N., Peile, E. \& Illing, J. (2012) Junior Doctors Prescribing: Enhancing their Learning in Practice, British Journal of Clinical Pharmacology, 73, 2, pp.194-202.

Ryan, C., Ross, S., Davey, P., Duncan, E.M., Francis, J.J., Fielding, S., Johnston, M., Ker, J., Lee, A.J., MacLeod, M.J. \& Maxwell, S. (2014) Prevalence and causes of prescribing errors: the PRescribing Outcomes for Trainee Doctors Engaged in Clinical Training (PROTECT) study, PloS one, 9, 1.

Tully, M.P. (2012) Prescribing Errors in Hospital Practice, British Journal of Clinical Pharmacology, 74, 4, pp. 668-675.

Van Doormaal, J.E., van den Bemt, P.M., Mol, P.G.M., Zaal, R.J., Egberts, A.C., HaaijerRuskamp, F.M. \& Kosterink, J.G.W. (2009) Medication Errors: The Impact of Prescribing and Transcribing Errors on Preventable Harm in Hospitalised Patients, BMJ Quality \& Safety, 18, 1, pp. 22-27. 